\title{
Fatigue Detection Using Computer Vision
}

\author{
Mitesh Patel, Sara Lal, Diarmuid Kavanagh, and Peter Rossiter
}

\begin{abstract}
Long duration driving is a significant cause of fatigue related accidents of cars, airplanes, trains and other means of transport. This paper presents a design of a detection system which can be used to detect fatigue in drivers. The system is based on computer vision with main focus on eye blink rate. We propose an algorithm for eye detection that is conducted through a process of extracting the face image from the video image followed by evaluating the eye region and then eventually detecting the iris of the eye using the binary image. The advantage of this system is that the algorithm works without any constraint of the background as the face is detected using a skin segmentation technique. The detection performance of this system was tested using video images which were recorded under laboratory conditions. The applicability of the system is discussed in light of fatigue detection for drivers.
\end{abstract}

Keywords-Face detection, eye blink detection, fatigue, computer vision.

\section{INTRODUCTION}

$\mathbf{F}$ ATIGUE has become one of the major causes of road accidents and has gained significant importance on the agenda of road safety. Fatigue as defined by Brown in 1994 is a disinclination to continue performing the task, and involves an impairment of human efficiency when work continues after the person becomes fatigued [1]. Driver fatigue is associated with reduced mental alertness which impairs performance of a range of cognitive and psychomotor tasks including driving [2]. A survey done in the U.S.A. by The National Highway Traffic Safety Administration (NHTSA) estimated that in 1996 there were 56,000 sleep related road crashes in the U.S.A. [3]. Another survey done it 2007 says that $18 \%$ of fatal accidents involved fatigue as the main factor. In Great Britain up to $20 \%$ of serious road accidents is caused due to fatigue [4]. Similarly, a survey done by the Road and Traffic Authority (Australia) states that in the year 2007, fatigue contributed to $20 \%$ of fatalities caused on road [5].

Fatigue measuring techniques can be broadly categorized into three types. The first set of techniques involves placing movement sensors on standard vehicle components like the steering wheel or gas pedal and analyzing the signals sent by these sensors [6]. In this work, the chaos theory was applied to explain the change of steering wheel motion. Takens' theory of embedding called attractor was used to find this strange trajectory of chaos motion of steering wheel [6]. This is more of a mechanical approach and the drivers need to adapt to the new system. This technique is not explored further as every

This work was supported under an Australian Research Council grant (ARC Linkage grant No. LP0562407)

M. Patel, S. Lal, and D. Kavanagh are associated for School of Medical and Molecular Biosciences, University of Technology, Sydney (UTS), NSW 2007, Australia (e-mails: miteshkumar.n.patel@eng.uts.edu.au, Sara.Lal@uts.edu.au, Diarmuid.Kavanagh@uts.edu.au).

P. Rossiter is associated with Forge Group, 241 Broadway, NSW 2007 Australia (e-mail: pmr@forge.com.au). driver has a different way of using vehicle components and hence its applicability cannot be generalized.

The second set of technique involves measurement of physiological signals such as Heart Rate (ECG), Electroencephalography (EEG) [7], and Electromyography (EMG). Heart rate variability provides a passive means to quantify drowsiness physiologically [8]. It is defined as the measure of variation in heart beats and is calculated by analyzing the time series of beat to beat intervals (i.e., the R-R intervals). This is a non-invasive technique, although it has a drawback in terms of practicality as it requires the person to wear an EEG cap while driving which can be intrusive.

The third set of techniques involves computer vision systems that can detect and recognize different facial expressions and the changes in facial appearance that occur during fatigue states [9]. This method is also non-invasive and has the advantage of using computer vision techniques, and therefore being more amenable to use by the general public [9]. Computer vision techniques employed to detect fatigue have focused on analysis of blinks, head movements and facial expression such as mouth, yawning [7], [10] and nose wrinkles.

In this paper we aim to report about a first version of an eye blink detection system. The system is designed within the scope of the SMART DATA project, whose goal is to develop fatigue system using different parameters which include physiological signals, computer vision.

\section{RELATED WORK}

Recently many countries have noted the importance of improving driving safety. Developing vision based warning systems for drivers is an increasing area of interest.

Computer vision has gained a lot of importance in the area of face detection, face tracking, eye detection etc for various applications like security, fatigue detection, biometrics. This technique has gained importance due it is non-invasive nature. Proper face detection is one of the most important criteria in a vision based fatigue detection system as the accuracy of the entire method relies on the accuracy of face detection. Various face detection techniques have been developed by different researchers.

In 2000, Margrit and William developed a driver monitoring system using edge detection and template matching technique [11]. The face detection routine was developed based on statistical decision theory using bayes decision rule to estimate from the color of the pixel if it images a face. The estimator was trained using images that were known to contain faces to determine a priori probability distributions of skin color. The peculiarity of this method was that it minimized the average loss due to the classification decision by using the likelihood ratio. The algorithm was not fully error free and gave an accuracy of about $56 \%$. 
Another eye detection model was developed by Takehiro in 2002 [12] using an infrared light projection method. The eye area is extracted from the captured whole facial image using separability filter. This method did not gain much popularity as infrared light with a wavelength of $850 \mathrm{~nm}$ was projected in pulse form on the driver which was both harmful and could not be implemented realistically.

In 2004, Wen-Bing and his associates developed a face detection and eye tracking technique by converting images from RGB (Red, Green and Blue) colour space to HSI (Hue, Saturation and Intensity) colour space. This model minimizes the errors in the system that might arise due to different brightness levels of different videos [13]. The system gave an accuracy of $88.9 \%$ when it was tested with four video data sets.

An eye detection technique using Hough Transform has gained lot of importance as the accuracy level of using this technique is high [14]. The only drawback of this technique is its high computational requirement. In 2007, Marc and his collegues developed real-time eye blink detection with GPU-based SIFT tracking. In the scope of their work they used a Facial Action Coding System (FACS) that allows the decomposition of facial expression in terms of facial feature displacements [15].

In 2007, David and Lim developed an eye tracking system by combining the colour segmentation and Hough Transform technique [16]. In this technique the image was converted from RGB colour space to $Y C b C r$ ( $Y=$ luma component, $C b=$ Blue-difference Chroma component and $\mathrm{Cr}=$ Red-difference Chroma component) colour space. The value of $\mathrm{Cb}$ and $\mathrm{Cr}$ for skin lies in a specific range which was used to extract only the facial region from the entire image. Using masking the unwanted face features were filtered. Hough transform was used on this filtered image to trace circle of the eye.

\section{Why Eye Detection}

In this paper we propose a Driver Fatigue System which is based on assessing eye blink rate. Eye blink detection implies prior detection of the eyes in the image of the subject's face. The face is detected by developing a skin segmentation model using Gaussian distribution. The skin templates available from the Matlab face detect tutorial [17] were used to develop the Gaussian model. The model converted the skin template from RGB (Red Green Blue) colour space to $\mathrm{YCbCr}$ colour space and used the $C b$ and $C r$ chrominance value to develop the skin segmentation model. On extraction of the face, a binary image of the face was used to detect the eyes.

\section{Proposing the Driver Fatigue Detection SYSTEM}

The driver fatigue system uses a colour cam-corder manufactured by JVC (Everio Hybrid Series, Australia). The camcorder captures the video image which is used by the fatigue detection system. The frame rate of the cam-coder is set to 30 frames per second. A frame is one of the many single photographic images in a motion picture. The recorded video is imported into Matlab (version 2007b, U.S.A) for further analysis and fatigue detection. Matlab is used for the analysis of video data and design of the fatigue detection system.

The flow chart for the driver fatigue detection system is shown in Fig. 2.

The fatigue detection system imports the video data and converts it into frames which are still images. The face is detected and extracted from this image using the Gaussian skin distribution spectrum. Since the system is based on the eye blink rate we further extract the region of importance which is the eye region in our case. According to the normal position of the eye it is reasonable to assume that it would lie between the upper $1 / 6^{\text {th }}$ and $2 / 5^{\text {th }}$ part of the face [13]. The eye region image is then converted into a binary image by converting the image from RGB image to Gray scale image. The iris is extracted from the eye region using the fact that the iris is darker than the surrounding region. This feature of the eye is exploited in our algorithm to extract the eye regions.

\section{A. Face Detection}

The step towards developing a face detection routine is to develop a skin segmentation model which is based on Gaussian distribution. This skin segmentation model would differentiate the skin colour in the image. We developed a skin segmentation model using the skin templates obtained from the Matlab face detect tutorial [17]. The obtained skin templates were cropped same size and that only pure skin areas were included (excluding nose and lips as well). These templates were converted from RGB to $\mathrm{YCbCr}$ space. Equation (1) was used to convert the RGB space image to $Y C b C r$ space image. The luminance ' $Y$ ' is discarded and only $C b$ and $C r$ are used to develop the Gaussian model. The non Gaussian histogram of the chrominance distribution of $\mathrm{Cb}$ and $\mathrm{Cr}$ is shown in Fig 1(a).

$\left[\begin{array}{c}Y \\ C b \\ C r\end{array}\right]=\left[\begin{array}{c}16 \\ 128 \\ 128\end{array}\right]+\left[\begin{array}{ccc}65.481 & 128.553 & 24.966 \\ -37.797 & -74.203 & 112.000 \\ 112.000 & -93.786 & -18.214\end{array}\right]\left[\begin{array}{c}R \\ G \\ B\end{array}\right]$

The mean and covariance matrix were calculated for both $\mathrm{Cb}$ and $\mathrm{Cr}$ components for the skin templates obtained from [17] were used to generate the value for Gaussian distributor model. The value of mean $\mathrm{Cb}, \mathrm{Cr}$ and its covariance obtained from the skin templates were used as the reference mean and covariance to compute the skin livelihood image. Equation (2) is the formula for the Gaussian distribution which was used to obtain the skin likelihood image. The skin likelihood image will project the part of the image which has the maximum probability of having skin pixel in that area. This equation is also known as mahalanobis distance equation [17]. The Gaussian distribution computed using this equation is shown in Fig 1(b). It can be observed from the histograms that the Gaussian distribution smoothens the raw distribution of $\mathrm{Cb}$ and $C r$ and hence gives higher accuracy of face detection.

$$
f(C b, C r)=\frac{1}{\sqrt{2 \pi \sigma}} \exp \left\{\frac{-\left(C r-\mu_{1}\right)\left(C b-\mu_{2}\right)}{2 \sigma}\right\}
$$

where

$$
\mu_{1}: \text { mean of } C b
$$


a)

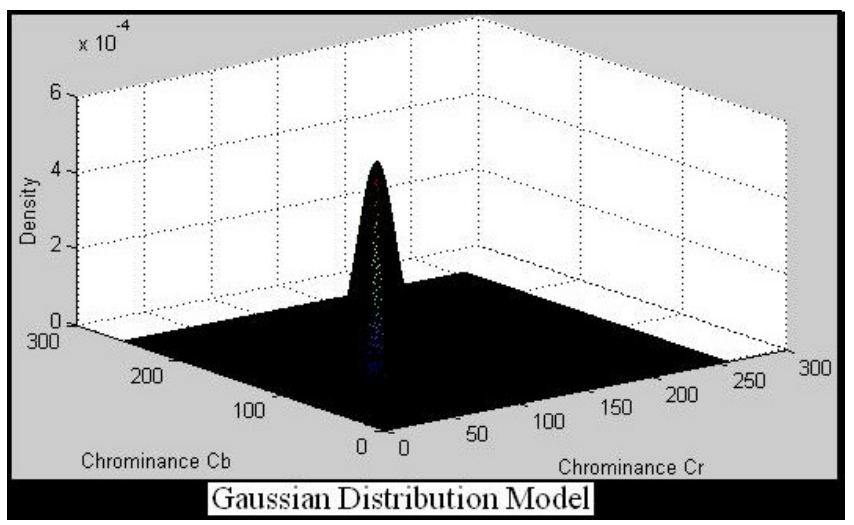

b)

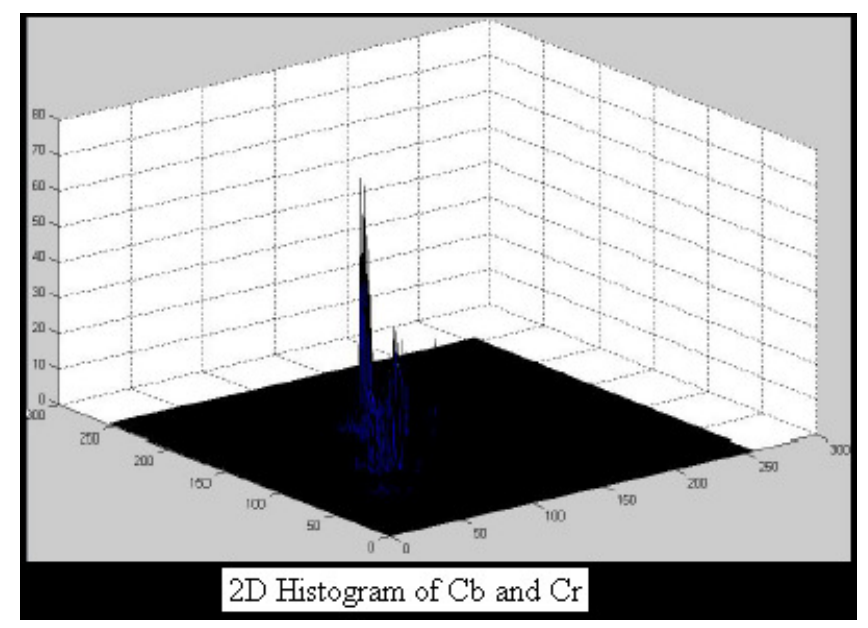

Fig. 1. Chrominance distribution using Gaussian model (a), raw chrominance distribution (b).

$\mu_{2}:$ mean of $\mathrm{Cr}$

$\sigma$ : variance of $\mathrm{Cb}$ and $\mathrm{Cr}$

The skin likelihood image obtained using equation (2) is normalized and is further used to generate a binary image. A binary image is generated from the skin likelihood image by using a threshold value so that pixels which have skin are valued one and pixels which do not have skin are valued zero. For our experiments the threshold values are not kept constant and can be varied with the individuals' complexion.

On obtaining the binary image, different morphological operations such as filling, erosion and dilation were applied so that we could separate skin areas which are loosely connected and also eliminate falsely detected pixels which might have filtered through while the binary image was being generated. Morphological operations were applied by using the erosion and dilation functions inbuilt in the Matlab software. Fig 3 shows the step-wise extraction of the facial section from the original image.

After detecting the face in the video image we applied horizontal and vertical projection techniques to extract the face from the entire image [13]. Horizontal and vertical projection is a technique where the algorithm will detect the border at

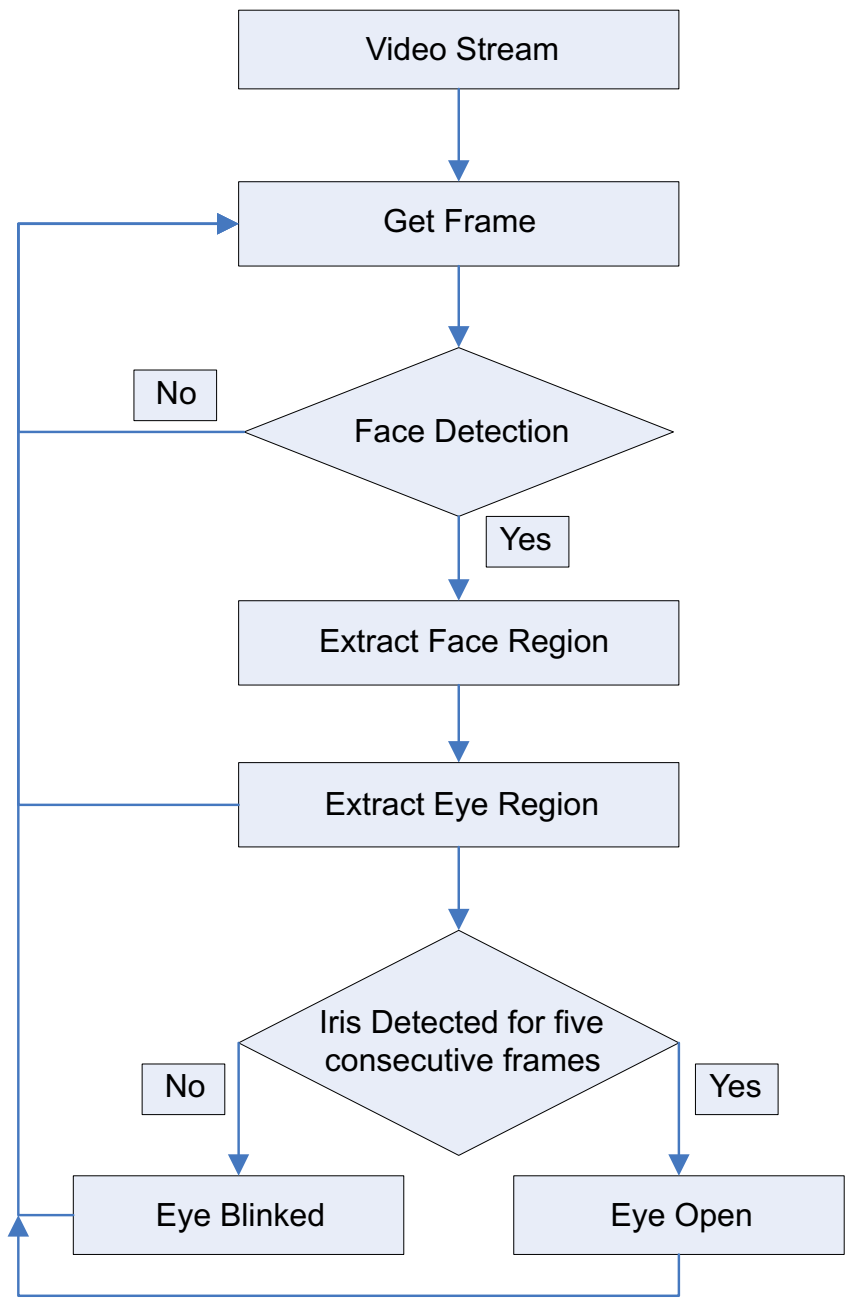

Fig. 2. Flow chart of driver fatigue detection system.

which there is a change in the binary value in the binary image. This change in value is detected as the boundary between which the face lies. Fig 4 shows cropping of the face from the entire image using the horizontal and vertical projection technique.

\section{B. Eye Detection}

After detecting the face the eye region can further be enhanced by removing unwanted facial features. The eye region is extracted from the face using the assumption that it lies between the upper $1 / 6^{\text {th }}$ and $2 / 5^{\text {th }}$ of the entire face region.

This eye region is further divided into individual eyes i.e. the left and the right eye. This is done by dividing the extracted eye region image vertically from the center. The iris is extracted by utilizing the fact that the iris region is the darker feature of the eye compared to the surrounding region. The extracted eye region was converted into a binary image so that the iris could be clearly visualized compared to the surrounding region. The iris was further extracted using the horizontal and vertical projection technique which was used to extract the initial face region. 


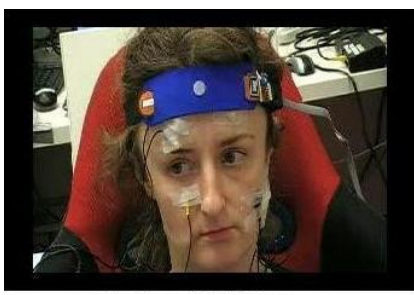

(a) Original RGB Image

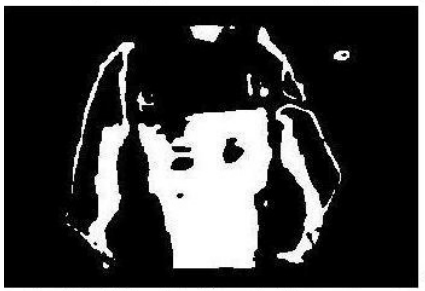

(c) Skin Segmented Image (Binary Image)

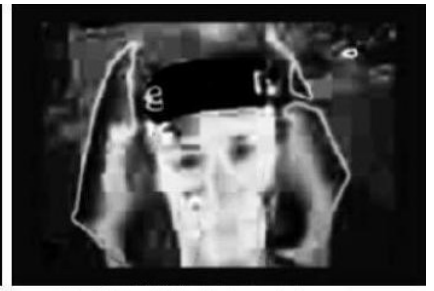

(b) Skin Likelyhood Image

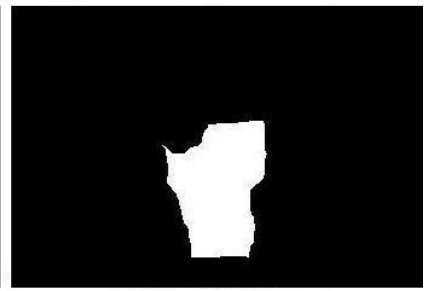

(d) Image After Erosion
Fig. 3. The original RGB (Red Green and Blue colour space) image(a), The skin likelihood image(b), The skin segmented image which is the binary image generated from the skin likelihood image(c), Image obtained after applying the morphological operations (Permission granted to reproduce the image)(d).

\section{Fatigue Detection}

The fatigue Detection system is based on assessing eye blink rate. The system detects an eye blink if it is not able to track the iris of the eye for 5 consecutive images. It will consider this pattern to be an eye blink [13].

Fig 4 shows the extraction of the face region from the entire image. The extracted eye in RGB is shown in Fig 5. Increase in blink rate denotes an increase in drowsiness. The proposed system works on the assumption that at the start, the driver is in an alert or normal state and records that blink rate as the base blink rate. To record this base blink rate the driver is asked to drive the simulator for 10 mins. This base blink rate is then used to compare the blink rate recorded every $30 \mathrm{sec}$. when the driver is driving with the simulator.

An increase in the blink rate by $10 \%$ of the base blink rate is detected by the system as a state of fatigue [13].

\section{EXPERIMENTAL RESULTS}

The software was tested with video images recorded from two volunteer participants. In most cases the software was able to detect the eye and hence the blink and the blink rate.

The major cause of concern could be false detection of the eyes. While extracting the eye, at times the algorithm confused the eyebrow and the eye as both appeared black when the images were converted into a binary image. Another factor which affected the accuracy was the eye lids. If the eyes were partially open then the algorithm had problems detecting the iris. Although sufficient measures were taken to get rid of this false detection, the system at present is not $100 \%$ error free. The accuracy of the developed system will be improved further when it will be validated with other video data in the future.

\section{Future WORK}

The proposed fatigue detection system has been developed using an image processing technique which uses a Gaussian

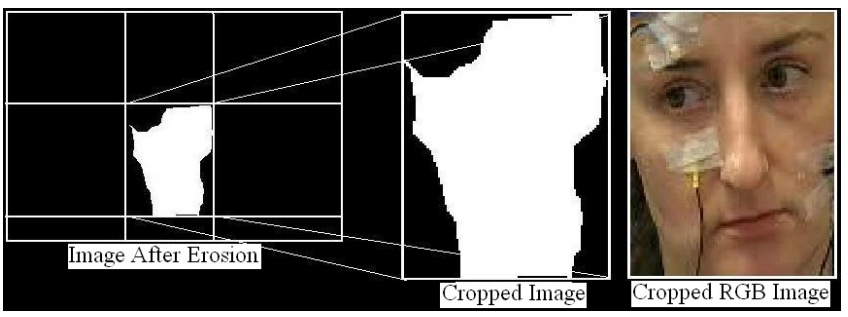

Fig. 4. Face detected cropped image (Permission granted to reproduce the image).

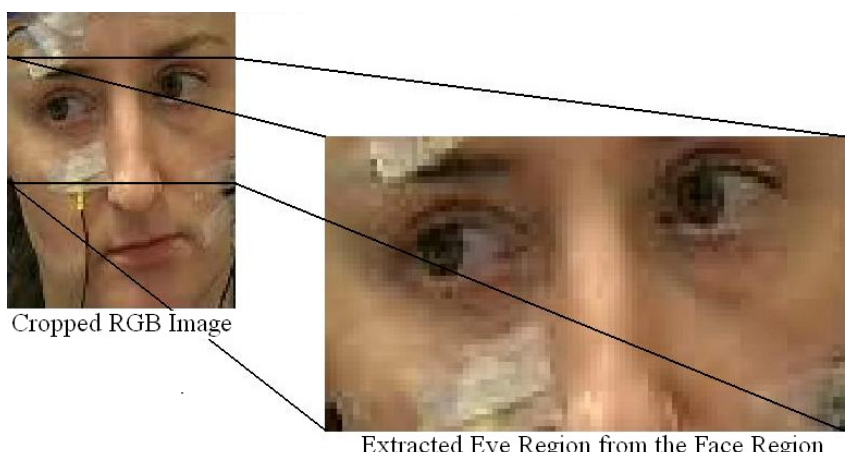

Fig. 5. Extracted Eye Region (Permission Granted to Reproduce the Image).

distribution model and horizontal and vertical projection techniques. The eye region can be determined quite reasonably using this technique.

The algorithm for the existing system has been entirely written in Matlab. Firstly this was due to Matlab's rich image processing tool box. Secondly as the video data has been analyzed for the first time and the algorithm under development is evaluated using different techniques. Though Matlab being a rich software source for analysis purposes, it has a major drawback with the processing time which is much longer when compared to other languages like $\mathrm{C}++$.

The current system tracks for the eye in every frame it processes which again increases processing time. The software can further be modified to have its own intelligence such that after it tracks the eye in the first frame it should search for the eye in the next frame taking into consideration the location of the eye in the previous frame. This will save a lot of processing time and lead to faster execution.

The current system is only capable of analyzing prerecorded video data which limits its use in real time. However, previously acquired data was essential to build and propose the fatigue detection system. Future work will be aimed at improving the accuracy of the fatigue detection system using previously acquired video image data from truck drivers. We also plan to implement a fatigue detection using head nodding. Further new work in the areas is aimed at constructing a system that can work in real time. We plan to use OpenCV, a well known $\mathrm{C}++$ library for computer vision. The real time system will help us to recod and analyze data simultaneously. 


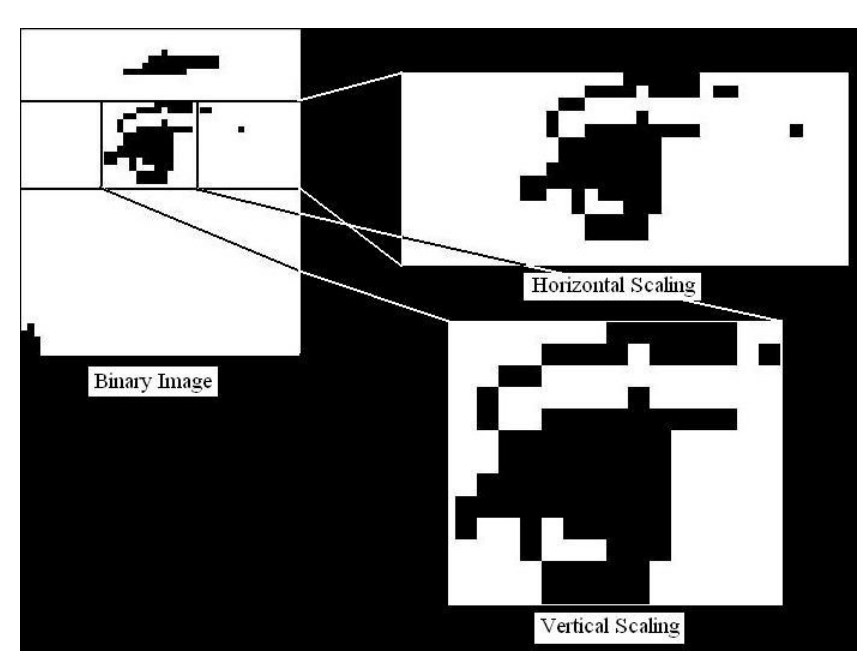

Fig. 6. Extraction of Iris.

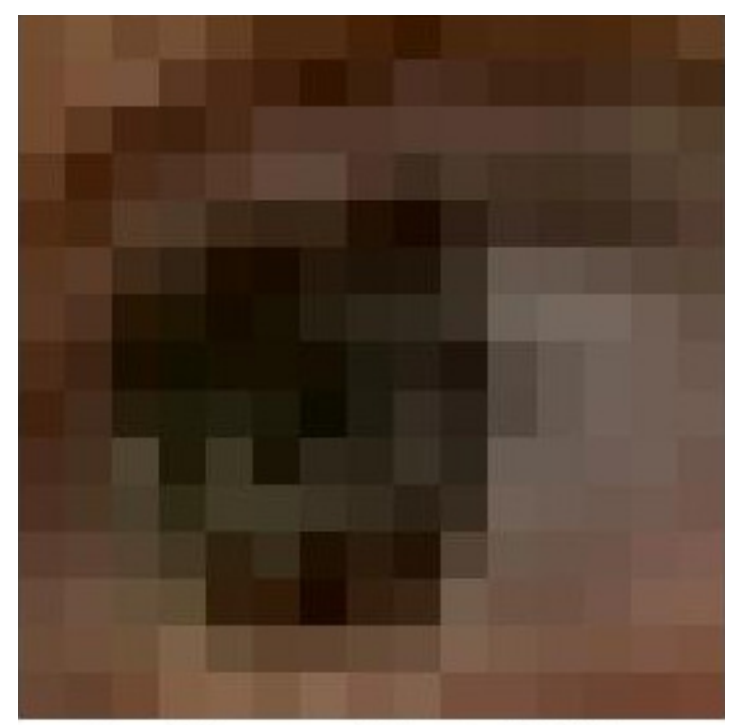

Final Cropped Eye in RGB

Fig. 7. Extracted Eye in RGB (Red, Green and Blue Colour Space).

\section{ACKNOWLEDGMENT}

The author would also like to acknowledge Monika Kent as a volunteer.

\section{REFERENCES}

[1] I. Brown, Driver Fatigue. Human Factors, 1994, vol. 36, pp. 298-314.

[2] A. M. Williamson, A. M. Feyer, and R. Friswell, "The impact of work practices on fatigue in long distance truck drivers," in Accident Analysis and Prevention,. Elsevier, 1996, vol. 28, pp. 709-719.

[3] S. Motorist, Driver Fatigue is an important cause of Road Crashes, Smart Motorist.

[4] Driver Fatigue and Road Accidents: A Literature Review and Position Paper, The Royal Society for the Prevention of Accidents, 2001

[5] Road and Traffic Authority (RTA) Annual Report, Sidney, 2008.

[6] Y. Takei and Y. Furukawa, "Estimate of driver's fatigue through steering motion," in International Conference on Systems, Man and Cybernetics, 2005, pp. 1765-1770.

[7] S. Lal and A. Craig, "Driver fatigue: Electroencephalography and psychological assesement," Psychology, vol. 39, pp. 313-321, 2002.

[8] L. Mulder, "Measurement and analysis methods of heart rate and respiration for use in applied envioroments," Biological Psychology, vol. 34, pp. 205-336, 1992.

[9] E. Vural, M. Cetin, A. Ercil, G. Littlewort, M. Barlett, and M. J. Drowsy, Driver Detection Through Facial Movement Analysis. Berlin/Heidelberg: Spinger, 2007.

[10] M. Saradadevi and P. Bajaj, "Driver fatigue detection using mouth and yowning analysis," International Journal of Computer Sciences and Network Security, vol. 8, pp. 183-188, 2008.

[11] M. Betke and W. J. Mullally, "Preliminary investigation of real-time monitoring of a driver in city traffic," in Proceedings of the IEEE Intelligent Vehicles Symposium, USA, 2000.

[12] T. Ito, S. Mita, K. Kozuka, T. Nakano, and S. Yamamoto, "Driver blink measurement by the motion picture processing and its application to drowsiness detection," in The IEEE 5th International Conference on Intelligent Transportation Systems, Singapore, 2002, pp. 168-173.

[13] W.-B. Horng, C.-Y. Chen, Y. Chang, and C.-H. Fan, "Driver fatigue detection based on eye tracking and dynamic template matching," in Proceedings of the 2004 IEEE International Conference on Netwroking, Sensing \& Control, 2004, pp. 7-12.

[14] T. D'Orazio, M. Leo, G. Cicirelli, and A. Distante, "An algorithm for real time eye detection in face images," in 17th International Conference on Pattern Recognition, 2004, pp. 278-281.

[15] M. Lalonde, D. Byrns, L. Gagnon, N. Teasdale, and D. Laurendeau, "Real-tiem eye blink detection with GPU-based SIFT tracking," in Computer and Robot Vision, 2007, p. 7.

[16] D. B. B. Liang and L. K. Houi, "Non-intrusive eye gaze direction tracking using color segmentation and hough transform," in International Symposium on Communications and Information Technologies, 2007, pp. 602-607.

[17] "AIT computer vision wiki matlab: Tutorial: detectface." 